\title{
Direct Monitoring of Intracellular Calcium Ions in Sea Anemone Tentacles Suggests Regulation of Nematocyst Discharge by Remote, Rare Epidermal Cells
}

\author{
PATRICIA MIRE-THIBODEAUX AND GLEN M. WATSON \\ Department of Biology, The University of Southwestern Louisiana, Lafayette, Louisiana 70504-2451
}

\begin{abstract}
In tentacles of sea anemones, cnidocytes and adjacent supporting cells are believed to be independent receptor-effector complexes that regulate nematocyst discharge in response to exogenous $\mathrm{N}$-acetylated sugars. When sugar chemoreceptors on supporting cells are activated, nematocyst discharge is two- to threefold greater than discharge without chemosensitization. To examine the role of $\mathrm{Ca}^{2+}$ as a second messenger in chemodetection of sugars, we used fluo-3 to monitor $\mathrm{Ca}^{2+}$ levels in epidermal cells of intact anemone tentacles. Certain epidermal cells exhibit relatively high $\mathrm{Ca}^{2+}$ both with and without chemosensitization. With chemosensitization, a twoto threefold increase occurs in the abundance of relatively rare cells exhibiting the highest $\mathrm{Ca}^{2+}$ levels. Timecourses depicting abundances of these rare cells in chemosensitized specimens show positive correlations to timecourses for nematocyst discharge from chemosensitized specimens and for labeling of chemoreceptors. Cnidocyte/supporting-cell complexes discharging nematocysts are about three times more abundant than the rare cells exhibiting the highest intracellular $\mathrm{Ca}^{2+}$ levels. One interpretation of these data is that the $\mathrm{Ca}^{2+}$-dependent regulation of nematocyst discharge occurring with chemosensitization involves intense $\mathrm{Ca}^{2+}$ signaling by remote, rare cells. This interpretation is inconsistent with the current model that portrays cnidocyte/supporting-cell complexes as independent effectors of nematocyst discharge.
\end{abstract}

\section{Introduction}

Sea anemones and other cnidarians, such as jellyfishes, hydras, and corals, are simple animals that use complex

Received 15 April 1993; accepted 15 September 1993. secretory products called nematocysts for prey capture, defense, and aggression. Nematocysts, consisting of intracellular capsules containing highly folded, eversible tubules, are produced in and deployed from effector cells called cnidocytes (Skaer and Picken, 1965). Cnidocytes within tentacles discharge nematocysts in response to appropriate chemical and mechanical stimulation (Pantin, 1942b; Thorington and Hessinger, 1988a, b). Discharge involves a rapid and forceful eversion of the tubule (Holstein and Tardent, 1984). Depending on the type of nematocyst, the everting tubule may penetrate the target to release potent toxins or adhere to the surface of the target (Mariscal, 1974). Although cnidocytes are abundant in tentacles, each nematocyst can be used only once (Robson, 1988). Thus, it is advantageous for the animals to control nematocyst discharge, and several cnidarians have been found to exercise such control (Pantin, 1942b; Williams, 1968; Lubbock, 1979; Thorington and Hessinger, 1988a).

In tentacles of the sea anemone Haliplanella luciae, nematocyst discharge is regulated, at least in part, by receptor-effector complexes composed of cnidocytes and supporting cclls (Watson and Hessinger, 1989). According to the current model, chemoreceptors on supporting cells detect substances derived from suitable target organisms and sensitize mechanoreceptors to trigger discharge upon physical contact between the tentacles and the target organism. Of the chemical stimuli that modulate discharge, most is known about the effects of $\mathrm{N}$-acetylated sugars. Cnidocyte/supporting-cell complexes (CSCCs) discharge nematocysts in response to both free and conjugated $\mathrm{N}$ acetylated sugars. The response is dose-dependent: at optimal doses of the sugars $\left(10^{-7} \mathrm{M}\right.$ for Haliplanella $)$, a twoto threefold increase in discharge is detected compared to 
that in seawater alone (Thorington and Hessinger, 1988a, b; Watson and Hessinger, 1987, 1989). The supportingcell chemoreceptors are cycled via receptor-mediated endocytosis so that receptor number changes over time (Watson and Hessinger, 1987, 1989). Interestingly, nematocyst discharge to optimal doses of sugars also changes over time and exhibits a strong positive correlation to temporal changes in numbers of activated surface chemoreceptors.

Relatively little is known about the specific cellular interactions or intracellular processes regulating nematocyst discharge. To investigate $\mathrm{Ca}^{2+}$ as a second messenger in the chemodetection of sugars, we used fluo- 3 to monitor $\mathrm{Ca}^{2+}$ levels in intact tentacles exposed to optimal levels of sugar and to seawater alone.

\section{Materials and Methods}

\section{Reagents}

Bovine submaxillary mucin, type I, and rhodaminedextran $10 \mathrm{~s}(\mathrm{MW}=9000)$ were obtained from Sigma; calcium ionophore 4-bromo A23187, fluo-3 AM, and pluronic acid F-127 were obtained from Molecular Probes. $\mathrm{Ca}^{2+}$-free artificial seawater $\left(\mathrm{Ca}^{2+}\right.$-free $\left.\mathrm{ASW}\right)$ was prepared in deionized water as follows: $\mathrm{NaCl}(448 \mathrm{mM}), \mathrm{KCl}$ (9 mM), $\mathrm{MgCl}_{2}-6 \mathrm{H}_{2} \mathrm{O}(23 \mathrm{mM}), \mathrm{MgSO}_{4}-7 \mathrm{H}_{2} \mathrm{O}(25 \mathrm{~m} M)$, $\mathrm{NaHCO}_{3}(2 \mathrm{~m} M)$, and EGTA (2 mM) (Sigma). Magnesium/seawater (MgSW) was prepared by dissolving $0.6 \mathrm{M}$ $\mathrm{MgCl}_{2}-6 \mathrm{H}_{2} \mathrm{O}$ in deionized water and then diluting 1:1 with natural seawater.

\section{Animal maintenance}

Monoclonal specimens of the sea anemone Haliplanella luciae were cultured in Pyrex dishes containing natural seawater at $32 \%$ and held at $22-24^{\circ} \mathrm{C}$. The anemones were maintained on a 12:12 photoperiod and fed brine shrimp nauplii (San Francisco Bay) twice weekly. Experiments were performed about $72 \mathrm{~h}$ after feeding.

\section{Microscopy}

Anemones were prepared for microscopy by anesthetizing them for $60 \mathrm{~min}$ in one of two media. MgSW was used for experiments in which fluo fluorescence and autofluorescence were monitored in the presence of external $\mathrm{Ca}^{2+} \cdot \mathrm{Ca}^{2+}$-free ASW was used when $\mathrm{Ca}^{2+}$-independent fluo fluorescence was monitored. These solutions served as media during labeling, washing, and viewing of the specimens.

For experiments involving fluo, whole animals were incubated in a $20-\mu M$ fluo-3 AM solution for $40 \mathrm{~min}$ at $23^{\circ} \mathrm{C}$ and then washed twice for $10 \mathrm{~min}$ each. Tentacles were excised, placed on glass coverslips, and secured with glass micropipettes (tip diameters of $<1 \mathrm{~mm}$ ) by applying gentle suction with microinjectors (Narashigi). The tentacles were viewed with an IMT2 Olympus inverted microscope using a $40 \times$ SPlan objective (n.a. $=0.9$ ). The fluorescence light source used was a $100-W$ halogen lamp set at $12 \mathrm{~V}$ with a Leitz power supply. This light source caused virtually no detectable radiation damage to the specimens and produced negligible autofluorescence. In contrast, preliminary work using a $100-\mathrm{W}$ mercury lamp showed extensive radiation damage to specimens (the tissue rapidly deteriorated) and produced more autofluorescence. Images were taken with a coolcd CCD camera $\left(-15^{\circ} \mathrm{C}\right.$ typical, $192 \mathrm{H} \times 165 \mathrm{~V}$ pixel array; Electrim Inc.), exposure time $20 \mathrm{~s}$, and analyzed using VGACAM (Electrim Inc.) and IPPLUS software (Media Cybernetics).

For studies of morphology and organization of cells in intact tissue, excised tentacles were viewed with DIC optics (DPlanApo100UV objective [n.a. $=1.30]$ and BHS-2 APlanat Achromat DIC condenser [n.a. $=1.4$ ], modified to fit the IMT-2 microscope). Video images were digitally processed using a Hamamatsu Argus 10 processor. Video recordings $(8 \mathrm{~mm})$ were made while focusing through the tissue to facilitate tracking of cell processes through optical sections. From these recordings, images were captured, enhanced, and analyzed using Image 1 software and hardware. To permit visualization of supporting cells and sensory cells, tentacles were depleted of cnidae by exposure to a hypotonic seawater solution prepared by diluting MgSW 1:1 with distilled water. The osmotic stress promotes cnida extrusion from tissue while leaving other cells intact for several hours.

\section{Timecourse experiments for intracellular $\mathrm{Ca}^{2+}$}

Timecourse experiments were performed with unlabeled or fluo-labeled tentacles in which fluorescence was monitored in epidermal cells following exposure either to $\mathrm{MgSW}$ (controls) or to MgSW containing optimal doses $\left(10^{-7} M\right)$ of mucin, a glycoprotein with many terminal $\mathrm{N}$-acetylated sugars (mucin treatment). For each experiment, a single tentacle was excised, secured, and allowed to recover for $10 \mathrm{~min}$ in washing medium. This solution was then replaced with a fresh drop of medium appropriate for the experimental condition, and areas of the tentacle epidermis were imaged at 5-min intervals for $60 \mathrm{~min}$. Specimens remained in the dark between exposures. A single image was made at each of the 13 timepoints.

\section{Ascertaining $\mathrm{Ca}^{2+}$-dependent fluorescence and testing effects of mucin}

To verify $\mathrm{Ca}^{2+}$-dependent fluo fluorescence in tentacles and to test the effects of mucin on $\mathrm{Ca}^{2+}$-dependent fluo fluorescence, four timecourse experiments were performed. To determine the intensity of autofluorescence, 
unlabeled tentacles were imaged. To determine the intensity of fluo fluorescence in the absence of $\mathrm{Ca}^{2+}$, fluo-labeled tentacles were incubated for $1 \mathrm{~h}$ in $10 \mu \mathrm{M}$ calcium ionophore, 4-Bromo $\mathrm{A} 23187$, prepared in $\mathrm{Ca}^{2+}$-free ASW and then exposed to $10^{-7} \mathrm{M}$ mucin prepared in $\mathrm{Ca}^{2+}$-free ASW prior to imaging. To examine $\mathrm{Ca}^{2+}$-dependent fluo fluorescence and the effect of mucin on $\mathrm{Ca}^{2+}$-dependent fluo fluorescence, labeled tentacles were imaged in MgSW alone or after exposure to $10^{-7} \mathrm{M}$ mucin. For each of these experiments, gray-level values were recorded (from 0 to 255) for the 10 most intensely fluorescent epidermal cells at each timepoint. Means were calculated from these values and then averaged from replicate experiments to derive a mean maximum fluorescence value for each timepoint. Such derived values provided an index of the fluorescence intensity, over time, for the brightest cells per treatment, taking into account day-to-day variation between experiments.

\section{Correlation to chemoreceptor activity}

Preliminary observations suggested that fluo-labeled tentacles showed an increase in abundance of the brightest epidermal cells upon exposure to mucin. This phenomenon was examined in greater detail by counting the brightly fluorescent cells in fluo-labeled tentacles in either MgSW alone or with the addition of $10^{-7} M$ mucin. A brightly fluorescent cell (BFC) was defined as one having a gray-level value at least 20 levels higher than the mean maximum fluorescence of the tentacle. This mean value was calculated by averaging gray-level values for the 10 most intensely fluorescent epidermal cells for each image acquired during a timecourse experiment. This step normalized data against day-to-day variations in labeling intensity. A gray-level value of 20 was selected for defining BFCs because it approximated the mean standard deviation for the mean maximum fluorescence of the tentacles used for the five replicate experiments. Data indicating number of BFCs were pooled from five replicate experiments for each condition (MgSW alone or $10^{-7} \mathrm{M}$ mucin in $\mathrm{MgSW}$ ).

\section{Timecourse experiment for nematocyst discharge}

To measure nematocyst discharge in the absence of $\mathrm{N}$ acetylated sugars, timecourse experiments were performed using the procedure described by Watson and Hessinger (1989). These results were compared to previously published timecourses for discharge following exposure to sugars (Watson and Hessinger, 1989) and to timecourses depicting the abundance of BFCs in both the absence and presence of $\mathrm{N}$-acetylated sugars.

Briefly, anemones were removed from the mass culture and placed in small petri dishes filled with natural seawater. After a recovery of 3-4 h, the medium was replaced with fresh natural seawater. After $10 \mathrm{~min}$ in the fresh medium, tentacles were touched with gelatin-coated test probes. Following glutaraldehyde fixation, the probes were prepared as wet mounts and the microbasic p-mastigophore nematocysts discharged into the gelatin were counted. Counts were recorded from a single field of view (10X oculars, $40 \times$ objective, and phase contrast optics) for each probe. For each timepoint, four replicate probes were used: one probe for each of four anemones. A mean discharge value \pm standard error was calculated by averaging data from two separate experiments for each timepoint.

\section{Identification of cells involved}

Supporting cells were identified by exploiting their ability to endocytose. Fluo-labeled tentacles were incubated in a $0.1 \%$ dextran-rhodamine/seawater solution for $15 \mathrm{~min}$, rinsed quickly, and viewed immediately. Whereas fluo-3 AM is cell permeant, dextran-rhodamine is cell impermeant but may enter cells by endocytosis. With free $\mathrm{Ca}^{2+}$, fluo-3 has an excitation maximum at $506 \mathrm{~nm}$, an emission maximum at $526 \mathrm{~nm}$, and negligible fluorescence above approximately $600 \mathrm{~nm}$ (Minta et al., 1989). Dextran-rhodamine has an excitation maximum at $570 \mathrm{~nm}$ and an emission maximum at $590 \mathrm{~nm}$ (Molecular Probes, Inc.). Each of the two indicators was visualized with optics to optimize excitation and emission of the desired indicator while prohibiting significant, coincidental excitation and emission of the other indicator (for fluo3, a BP-490 excitation filter that permits transmittance between 450 and $500 \mathrm{~nm}$; for dextran-rhodamine, a BP$545+$ supplemental exciter filter EO-530 that permits transmittance between 500 and $570 \mathrm{~nm}$ and an additional R-610 barrier filter that prevents transmission of emitted light below approximately $600 \mathrm{~nm}$ ). Because supporting cells are the only epidermal cells known to endocytose (Watson and Hessinger, 1987, 1989), paired images of double-labeled tentacles were used to determine which of the cells exhibiting fluo fluorescence were likely to be supporting cells.

\section{Ratio of BFCs to nematocysts discharged}

Specimens were exposed to seawater alone or to seawater containing $10^{-7} \mathrm{M} \mathrm{N}$-acetylneuraminic acid (NANA) for $10 \mathrm{~min}$, then touched with gelatin-coated test probes. The average density of BFCs imaged (see above) in the epidermis of intact tentacles $(n=5)$ was compared with the density of nematocysts and other cnidae discharged into test probes $(n=10)$. We estimated the area of the tentacle epidermis imaged to be $2112 \mu \mathrm{m}^{2}$, half the total field of view of the camera. Areas on test probes subjectively evaluated to have the greatest number of nematocysts were scored. Microbasic p-mastigophore ne- 


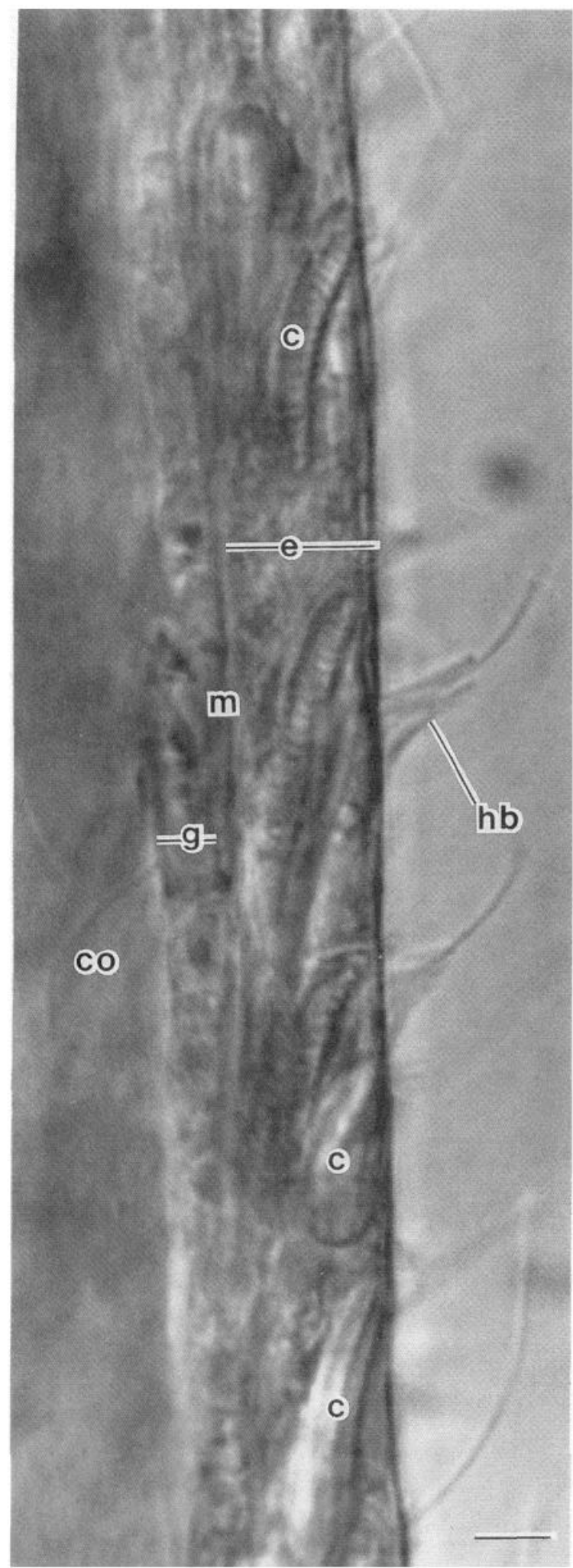

Figure 1. DIC photomicrograph of sea anemone tentacle. The epidermis (e), containing cnidocyte/supporting-cell complexes (CSCCs) and sensory-cell/supporting-cell complexes (SNSCs), is separated from the gastrodermis $(\mathrm{g})$ by the mesoglea $(\mathrm{m})$. Cnidae within cnidocytes $(\mathrm{c})$ of CSCCs are visible within the epidermis. Hair bundles (hb) at the apices matocysts, spirocysts, and basitrich nematocysts were counted on the test probes within a rectangular reticule measuring $38,880 \mu \mathrm{m}^{2}$. The mean number of nematocysts and other cnidae counted was divided by the mean number of BFCs to give a ratio. This value was multiplied by 0.054 to correct for the difference between the areas of the tentacle sampled for discharge and imaged for fluo fluorescence.

\section{Results}

\section{Morphology and organization of cells}

Sea anemone tentacles are composed of two tissue layers separated by a mostly acellular matrix called the mesoglea (Fig. 1). The epidermal layer lies adjacent to the external surface and the gastrodermal layer lines the internal cavity (the coelenteron).

Within the epidermis, supporting cells form complexes either with cnidocytes or with sensory cells $(\mathrm{CSCC}=$ cnidocyte/supporting-cell complex; SNSC = sensory-cell/ supporting-cell complex). In each of the complexes, a single cnidocyte or a single sensory cell is surrounded by several supporting cells. These supporting cells contribute small-diameter $(0.05-0.2 \mu \mathrm{m})$ stereocilia to a hair bundle that protrudes from the apical surface of the complex (Peteya, 1975). In the CSCC, the cnidocyte contributes a single kinocilium to the hair bundle (Mariscal et al., 1978). In the SNSC, the sensory cell contributes a single kinocilium and 5-10 large-diameter $(0.2-0.5 \mu \mathrm{m})$ stereocilia to the hair bundle (Peteya, 1975).

In CSCCs, cnidocytes are easily recognized by the presence of cnidae (microbasic p-mastigophores, basitrichs, or spirocysts); however, because cnidae are strongly birefringent, supporting cells are difficult to resolve clearly. Due to the extreme abundance of cnidae in tentacles, sensory cells and supporting cells of SNSCs are also difficult to visualize. Supporting cells and sensory cells can be better visualized in tentacles that have been depleted of cnidae (Fig. 2). From a lateral view, the apices of supporting cells are broad (approximately $10-\mu \mathrm{m}$ diameter) and triangular, and the bases gradually narrow into long tenuous processes that extend toward the mesoglea (Thibodeaux and Watson, 1991). Sensory cells have narrow apices (approximately $3-\mu \mathrm{m}$ diameter) and bases that are thought to terminate in synapses with the nerve net near the mesoglea

\footnotetext{
of CSCCs and SNSCs are apparent at the surface of the epidermis. (co $=$ coelenteron.) This photomicrograph was taken near the base of the tentacle where the cnidae are less abundant than at the tip, making the tissue layers easier to distinguish. This specimen was fully extended, causing the cnidae to become oriented obliquely to the epidermal surface. More commonly, cnidae are oriented perpendicular to the epidermal surface (see Fig. 2B). Scale bar $=5 \mu \mathrm{m}$.
} 

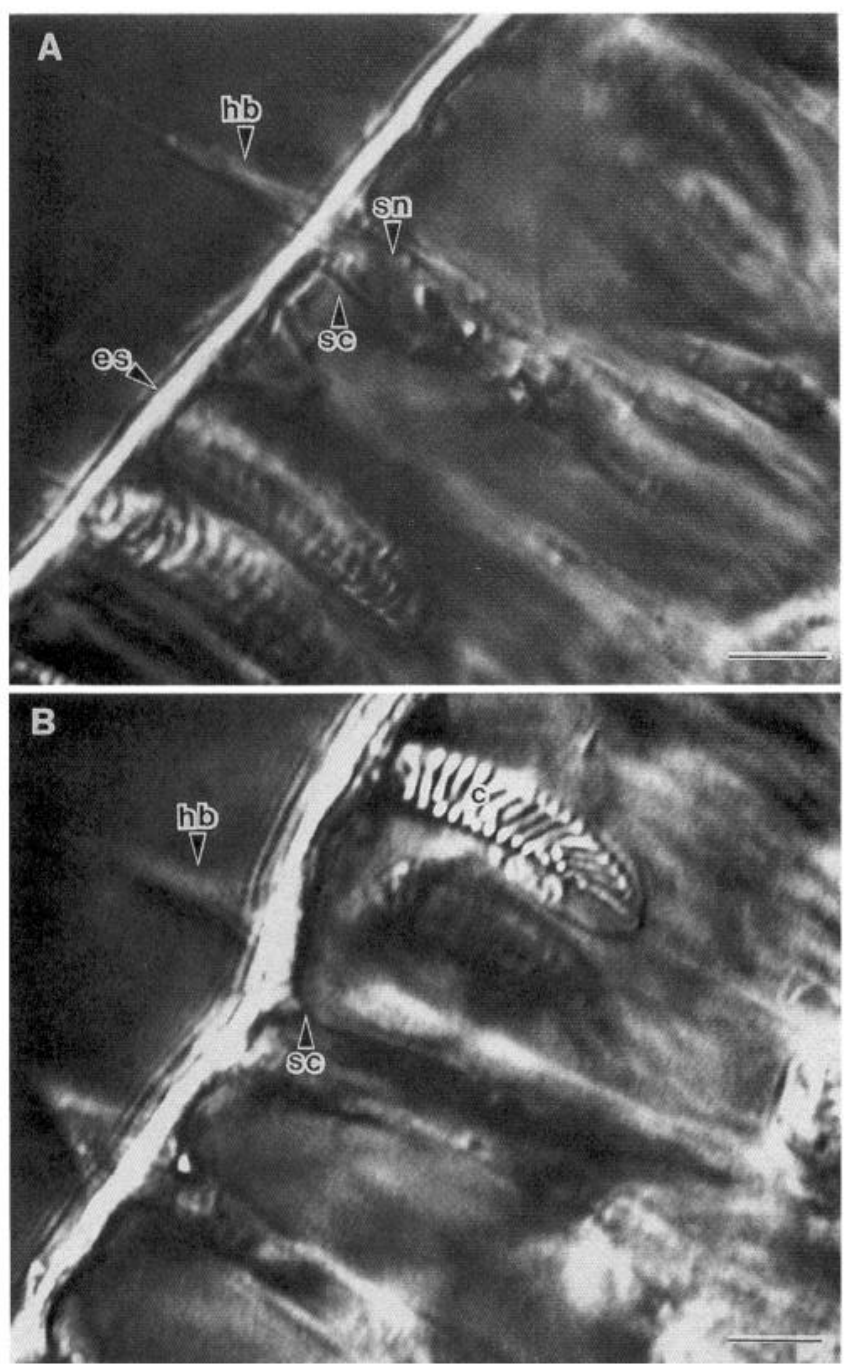

Figure 2. DIC video-enhanced photomicrographs of a sea anemone tentacle exposed to hypotonic medium to promote the extrusion of cnidae. Two optical sections of a SNSC: (A) The hair bundle (hb) extends into the seawater from the epidermal surface (es). Beneath the epidermal surface, the sensory cell (sn) of the SNSC and several supporting cells (sc) surrounding it extend toward the mesoglea located just beyond the border of the micrograph; (B) a deeper optical section of the same SNSC more clearly shows the triangular apex of one of the supporting cells (sc) of the SNSC. A cnidocyte (c) is in focus. Scale bar $=5 \mu \mathrm{m}$.

(Peteya, 1975). Although CSCC involvement in the regulation of nematocyst discharge has been well characterized (Watson and Hessinger, 1989, 1992), SNSC involvement, if any, is not known.

\section{Description of fluo fluorescence}

Fluo-labeled tentacles exhibited fluorescence both in the epidermis and in the gastrodermis (Fig. 3A). Whereas autofluorescence within the gastrodermis was so intense that it overwhelmed fluo fluorescence, autofluorescence within the epidermis did not significantly interfere with detecting $\mathrm{Ca}^{2+}$-dependent fluo fluorescence (see below). Only certain cells within the epidermis were fluorescent above autofluorescence levels; the fluorescence intensity of these cells varied somewhat, both in MgSW controls and in mucin-treated specimens. Most of the fluorescent cells occurred at the tentacle surface and had a triangular shape reminiscent of supporting cells. Less frequently, extremely bright cells were observed within the epidermis at the tentacle surface (Fig. 3A, B). The glare from the intense fluorescence made the morphology of these cells difficult to distinguish. The least common brightly fluorescent loci occurred deeper within the epidermis, often with fine processes extending toward both the epidermal surface and the mesoglea (Fig. 3A). It was not clear whether these bright loci constituted domains of cells extending to the epidermal surface or were contained within cells not in immediate contact with the surface.

\section{Identification of supporting cells}

To determine whether the triangular patterns of fluo fluorescence, described above, represented supporting cells, 30 paired images of tentacles double-labeled with fluo-3 and rhodamine-dextran were analyzed (Fig. 4). In comparisons of 380 cells, there was $80 \%$ agreement between cells endocytosing rhodamine-dextran (Fig. 4A) and those exhibiting $\mathrm{Ca}^{2+}$-dependent fluo fluorescence (Fig. 4B). To determine autofluorescence ranges, fluorescence intensity of unlabeled tentacles was measured with both fluorescein and rhodamine optics; filters were used to prevent crossover excitation and emission of the two indicators (see Materials and Methods). Only cells exhibiting values higher than autofluorescence were used in the pairwise analysis.

\section{$\mathrm{Ca}^{2+}$-dependent fluo fluorescence and mucin effect}

Fluorescence intensity was measured for the 10 brightest epidermal cells per microscopic field at each timepoint. Mean values were calculated for each timepoint of each replicate experiment. The resulting means from these replicates, averaged to give mean maximum fluorescence values (see Materials and Methods), were compared for autofluorescence, $\mathrm{Ca}^{2+}$-independent fluo fluorescence, and $\mathrm{Ca}^{2+}$-dependent fluo fluorescence in $\mathrm{MgSW}$ controls and in mucin-treated specimens (Fig. 5). Statistical analyses (ANOVA) of the timecourse data (considering data sets consisting of between 10 and 13 timepoints; Fig. 5) indicated heterogeneity among the means for the treatments $\left(p=2.45 \times 10^{-23}\right)$. Post hoc comparisons (Tukey's LSD) revealed that $\mathrm{Ca}^{2+}$-dependent fluo fluorescence was significantly greater in mucin treatment than in $\mathrm{MgSW}$ controls $(p=.02)$. Additionally, autofluorescence and $\mathrm{Ca}^{2+}$. independent fluo fluorescence were comparable to each 

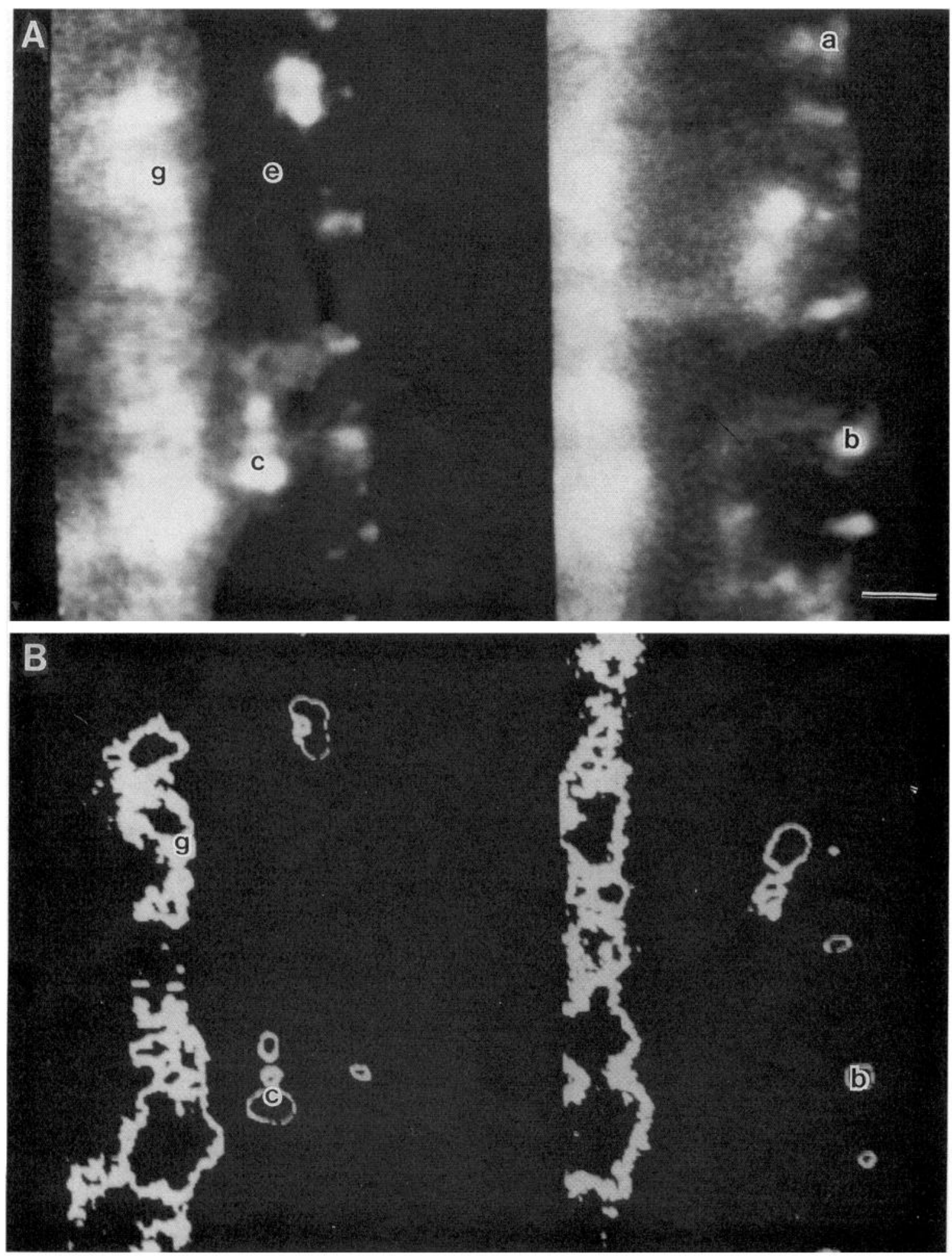

Figure 3. Cooled CCD photomicrographs of fluorescence from two fluo-labeled tentacles. (A) Several cells within the epidermis (e) are fluorescent, including triangular cells (a) exhibiting moderate fluorescence (see also Fig. 4A) and brightly fluorescent cells (BFCs) with intense fluorescence at (b) or beneath (c) the surface of the epidermis. The strongly autofluorescent gastrodermis ( $\mathrm{g}$ ) also is shown. Scale bar $=5 \mu \mathrm{m}$. (B) Contour plots depict gray values ranging from 121 to 131 for some of the brightest fluorescent cells shown in Figure 3A. Note that the triangular cell (a) in Figure 3A was too dim to be seen in Figure 3B. For presentation, contrast was linearly enhanced using IPPLUS software. 


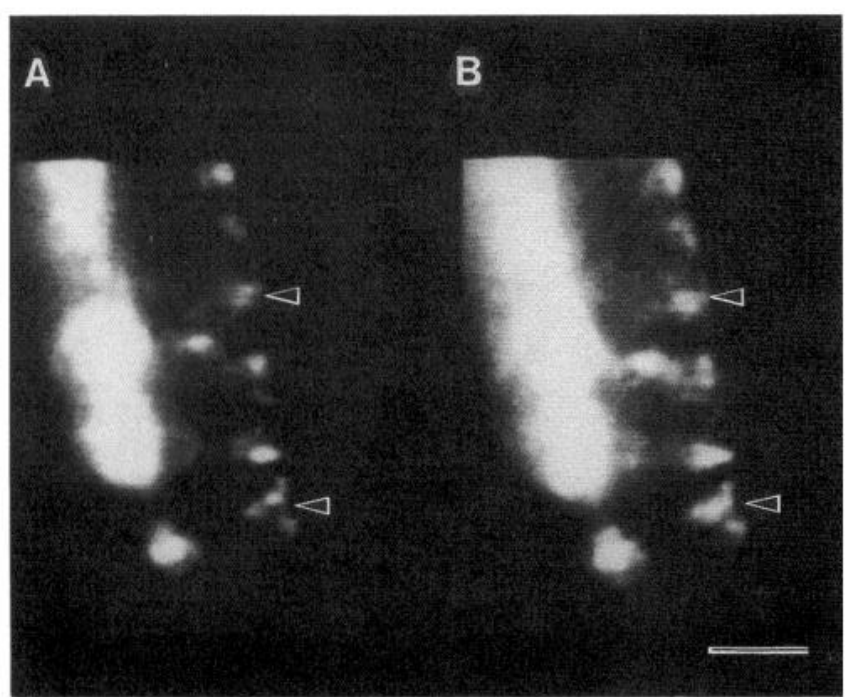

Figure 4. Cooled CCD paired photomicrographs of fluorescence from a fluo-labeled tentacle incubated in rhodamine dextran. (A) Rhodamine dextran fluorescence of the tentacle epidermis includes several bright loci. (B) Fluo fluorescence of the same region of the tentacle epidermis with several bright loci. Arrows indicate representative cells exhibiting fluorescence both for fluo and rhodamine-dextran. For presentation, contrast was linearly enhanced using IPPLUS software. Scale bar $=10 \mu \mathrm{m}$.

other but less than $\mathrm{Ca}^{2+}$-dependent fluo fluorescence ( $p$ $=.0002$ ).

\section{BFCs in mucin treatment versus $\mathrm{MgSW}$ controls}

To more precisely describe the difference in $\mathrm{Ca}^{2+}$-dependent fluo fluorescence attributable to the effects of mucin, total numbers of BFCs in mucin treatment were compared to BFCs in MgSW controls. Overall, BFCs were about twice as numerous in mucin-treated specimens as in control specimens. Statistical analysis (Student's $t$-test) comparing the mean number of BFCs per timepoint, computed by averaging data from five separate timecourses ( $n=650$ cells scored per treatment), indicated that the means for the two treatments were significantly different $(p<.05)$.

\section{Correlation of BFC abundance to chemoreceptor activity}

Timecourses depicting number of BFCs in both the absence and presence of mucin were compared to timecourses depicting numbers of nematocysts discharged in both the absence and presence of mucin, and to timecourses in which supporting-cell chemoreceptors were labeled with mucin-gold (Watson and Hessinger, 1987, 1989). A strong positive correlation was found between timecourses for nematocyst discharge from mucin-treated specimens (free mucin or gold-conjugated mucin) and timecourses for mucin-gold labeling at supporting cell surfaces (Fig. 6A and Table I). Furthermore, the timecourse data for BFCs in mucin-treated specimens were positively correlated to timecourses for both nematocyst discharge from mucin-treated specimens and surface chemoreceptor labeling with mucin-gold (Figs. 6B, C, and Table I). In contrast, data for BFCs in mucin treatment showed no correlation to data for BFCs in MgSW controls or to data for nematocyst discharge from specimens in seawater alone (Table I). Additionally, timecourse data for nematocyst discharge from specimens in seawater alone showed no correlation to data for BFCs in MgSW controls or to nematocyst discharge from specimens in mucin (Fig. 6D and Table I).

\section{Relative abundances of BFCs and nematocysts discharged}

The mean densities of BFCs and discharged nematocysts were compared (see Materials and Methods) to provide an index of the relative abundances of BFCs and responding CSCCs. In seawater controls, the ratio of BFCs to discharged nematocysts was 1 to 3.61 when only microbasic p-mastigophores were considered. In chemosensitized specimens, this ratio was 1 to 2.85 . Previous studies on nematocyst discharge have focused on microbasic pmastigophores (e.g., Watson and Hessinger, 1989) because they are easy to quantify. However, two other cnida types

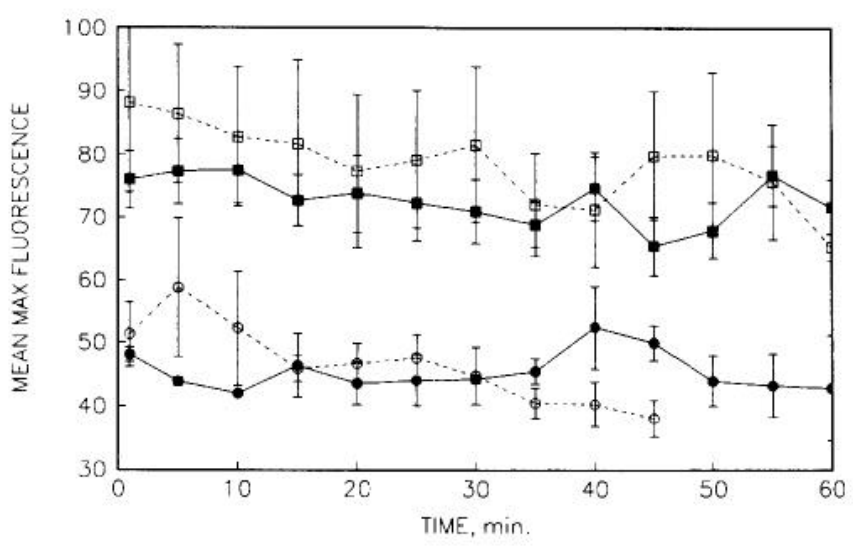

Figure 5. Timecourse of mean maximum fluorescence. Maximum gray values were recorded for the 10 brightest cells from digitized images collected at each time point. These values were averaged for each time point over five replicate experiments to give mean maximum fluorescence values. Timecourses for mean maximum fluorescence are shown (as gray-level values, \pm SEM) for autofluorescence (solid circles); and for fluo fluorescence in $\mathrm{MgSW}$ alone (solid squares); in MgSW containing $10^{-7} \mathrm{M}$ mucin (open squares); and in $\mathrm{Ca}^{++}$-free SW containing $10^{-7} M$ mucin after $1 \mathrm{~h}$ pretreatment of specimens in $10^{-5} M 4$-bromo A23187 prepared in $\mathrm{Ca}^{++}$-free $\mathrm{SW}\left(\mathrm{Ca}^{++}\right.$independent fluo fluorescence; open circles). The results of statistical analysis of the data, including ANOVA with LSD post hoc comparisons of the treatments (CSS Statistica software, Tulsa) are described in the text. In all cases, significant findings are reported in the text with a maximum $p$ value of .05 . 

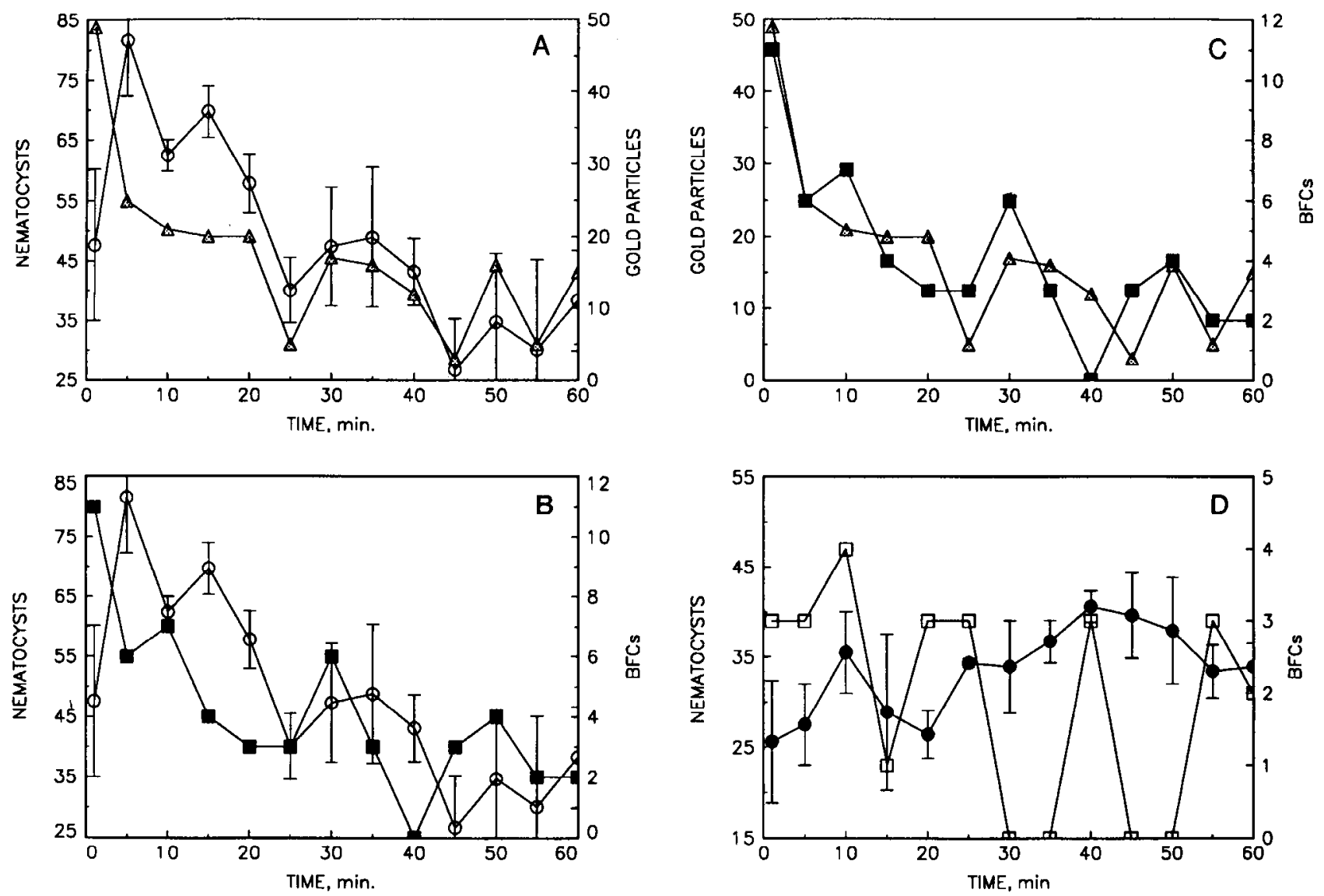

Figure 6. Timecourses for the abundance of mucin-gold particles observed at the apical surface of supporting cells, for the abundance of BFCs observed in the tentacle epidermis in both the presence and absence of $10^{-7} \mathrm{M}$ mucin, and for discharged nematocysts counted in test probes ( $\pm \mathrm{SEM}$ ) in both the presence and absence of mucin. To facilitate comparisons, timecourses are paired as follows: (A) mean nematocysts discharged within single microscopic fields $\left(0.16 \mathrm{~mm}^{2}\right)$ of test probes upon exposure to $10^{-7} \mathrm{M}$ mucin (open circles) and mucin gold particles counted at the apical surface of 25 supporting cells (speckled triangles, data from Watson and Hessinger, 1989); (B) mean nematocysts discharged after exposure to $10^{-7} \mathrm{M}$ mucin (open circles) and BFCs counted in tentacles after exposure to $10^{-7} \mathrm{M}$ mucin (solid squares); (C) mucin gold particles counted at the surface of supporting cells (speckled triangles) and BFCs counted upon exposure to $10^{-7} \mathrm{M}$ mucin (closed squares); and (D) mean nematocysts discharged into test probes in seawater alone (closed circles) and BFCs counted in tentacles exposed to $\mathrm{MgSW}$ alone (open squares). The results of statistical analysis of the data using Spearman rank correlation are shown in Table I.

occur in cnidocytes of tentacles: basitrichs and spirocysts. Although less is known about the processes involved in regulating discharge of these cnidae, they also occur in CSCCs in tentacles and routinely discharge into test probes. Spirocyst CSCCs respond to chemosensitization by $\mathrm{N}$-acetylated sugars (Thorington and Hessinger, 1990). When considering all of the types of cnida discharged, the ratio of BFCs to total responding CSCCs was 1 to 8.36 in seawater controls and 1 to 6.22 following chemosensitization.

\section{Discussion}

In this study, we used the $\mathrm{Ca}^{2+}$-dependent fluorescent dye fluo-3 to directly monitor intracellular $\mathrm{Ca}^{2+}$ levels in the epidermis of anemone tentacles (Fig. 3). Fluo-3 is only weakly fluorescent in the absence of $\mathrm{Ca}^{2+}$ and significantly increases in fluorescent intensity upon binding $\mathrm{Ca}^{2+}$. We show that intracellular $\mathrm{Ca}^{2+}$ levels are higher in tentacles exposed to optimal levels of $\mathrm{N}$-acetylated sugars (mucin treatment) than in tentacles exposed to seawater alone (MgSW controls) (Fig. 5). Furthermore, the abundance of cells exhibiting the highest $\mathrm{Ca}^{2+}$ levels, BFCs, is twoto threefold greater in mucin treatment than in $\mathrm{MgSW}$ controls. This increase in BFC abundance with mucin treatment is comparable in magnitude to the enhancement in nematocyst discharge that occurs upon exposure to mucin (Watson and Hessinger, 1987, 1989). Moreover, timecourses depicting BFC abundance in mucin-treated 
Table I

Positive correlations between fluorescence, nematocyst discharge, and chemoreccptor labeling under different conditions

\begin{tabular}{ll}
\hline \hline \multicolumn{1}{c}{$\begin{array}{c}\text { Significant correlations } \\
(p<.05)\end{array}$} & \multicolumn{1}{c}{$\begin{array}{c}\text { Insignificant correlations } \\
(p>.05)\end{array}$} \\
\hline BFCs mucin $v$ s. ND mucin & BFCs seawater $v$ s. BFCs mucin \\
BFCs mucin $v s$. ND mucin-gold & BFCs seawater $v$ s. ND seawater \\
BFCs mucin $v$ s. mucin-gold labeling & ND seawater $v s$. ND mucin \\
ND mucin $v$. mucin-gold labeling & \\
\hline
\end{tabular}

Correlation calculated using Spearman rank analysis of data from timecourse experiments.

BFCs, brightly fluorescent cells; ND, nematocyst discharge.

specimens are positively correlated to timecourses depicting nematocyst discharge to optimal levels of mucin and to timecourses for supporting-cell chemoreceptor labeling (Figs. 6A, B, C and Table I). Although these positive correlations do not prove causal relationships between BFC abundance and CSCC responsiveness, one plausible interpretation is that BFCs are somehow involved in the $\mathrm{Ca}^{2+}$-dependent regulation of nematocyst discharge from CSCCs upon chemosensitization with $\mathrm{N}$-acetylated sugars.

It is interesting, however, that in seawater alone, a few BFCs are observed in tentacles, and nematocyst discharge can be triggered at a baseline level (Fig. 6D). The cooccurrence of these two phenomena in the absence of $\mathrm{N}$ acetylated sugars may indicate that BFCs are also somehow involved in controlling nematocyst discharge in seawater alone. The duration of $\mathrm{Ca}^{2+}$ signaling by individual BFCs could not be determined by our methods, which incorporated relatively long exposures (20 s) and imaging of many different sites of the tentacle per timecourse experiment.

Results of morphological studies using DIC optics and of endocytosis experiments using dextran-rhodamine suggest that some cells exhibiting relatively high internal $\mathrm{Ca}^{2+}$, in both the presence and absence of mucin, are supporting cells (Fig. 4). However, it is not clear whether all cells exhibiting $\mathrm{Ca}^{2+}$-dependent fluorescence are supporting cells. This uncertainty is especially pronounced for the less common, brightest loci observed at the tentacle surface and deeper within the epidermis. In both cases, glare from intense fluorescence prevented our determining the precise morphology of the cells involved (Fig. 3).

Of the five cell types occurring at the tentacle surface (cnidocytes, supporting cells, sensory cells, two types of gland cell), we speculate that the extremely bright surface cells are components of CSCCs or SNSCs-either specialized cnidocytes and/or supporting cells, or sensory cells. Although these BFCs did not appear to possess cnidae, and therefore would not be cnidocytes, we cannot rule out the possibility that the intense fluorescence from these cells obscured cnidae within them. Supporting cells are the most abundant epidermal cells at the tentacle surfaces because several such cells make up each CSCC and each SNSC. CSCCs are highly abundant in tentacles. If supporting cells or cnidocytes of each activated CSCC were responsible for the intense fluorescence, one would expect a higher abundance of BFCs than was observed. Therefore, if these BFCs are supporting cells, or cnidocytes, or both, of CSCCs, we suggest they are specialized in some way so that only certain ones, among the many present in the tentacle epidermis, participate in intense $\mathrm{Ca}^{2+}$ signaling. These specialized CSCCs would be required to activate other CSCCs by a mechanism that would not involve extreme elevations in $\mathrm{Ca}^{2+}$ levels within the secondarily activated CSCCs.

SNSCs are less abundant than CSCCs. It is possible that BFCs are supporting cells, sensory cells, or both, of SNSCs. Sensory cells are reported to be involved in nervous integration in cnidarians (Bullock and Horridge, 1965; see also Shelton, 1982; Boothby and McFarlane, 1986; Westfall, 1988; Grimmelikhuijzen et al., 1990; McFarlane et al., 1990). In anemone tentacles, the sensory cell is thought to be a bipolar neuron in the tentacle epidermis with a narrow dendrite that terminates apically in a cluster of large stereocilia and basally in a long, tenuous axon that connects to the nerve net (Peteya, 1975; van Marle, 1990). The location and morphology of the bright loci deeper within the epidermis suggest that these BFCs could be neurons or basal domains of surface epidermal cells. These loci occurred in the vicinity of the nerve net and commonly appeared to have fine processes extending toward the surface or mesoglca. Although all threc types of cells proposed as candidates for surface BFCs (cnidocytes, supporting cells, and sensory cells) have been implicated as connecting to the nerve net, the evidence appears to be strongest for sensory cells (Westfall, 1973; Peteya, 1975; Bigger, 1982; van Marle, 1990).

The independent effector hypothesis, the classical explanation for the regulation of nematocyst discharge, portrays the cnidocyte as responding directly to mechanical and chemical stimuli by triggering discharge. In this view, the cnidocyte is both a receptor and effector cell acting independently of any nervous intervention (Parker, 1916, 1919; Parker and Van Alstyne, 1932; Pantin, 1942a, b; Ewer, 1947). Later interpretations of the hypothesis allow for some nervous intervention either to reduce or to increase discharge, depending on the physiological state of the animal. For example, in experiments assaying nematocyst discharge immediately following treatment, certain physically agitated anemones discharge more nematocysts and spirocysts than undisturbed anemones (Conklin and Mariscal, 1976). In this revision of the hypothesis, the nervous system is viewed as modulating the 
overall responsiveness of the cnidocytes, which still exercise self-regulation of discharge.

More recent evidence indicates that chemoreceptors regulating discharge are located on supporting cells, and not on cnidocytes. These results have prompted another revision of the hypothesis, which suggests that discharge is regulated by complexes consisting of cnidocytes and the supporting cells surrounding the cnidocytes (CSCCs). According to this model, the CSCCs act as independent effector complexes with the supporting cells detecting chemical stimuli, such as $\mathrm{N}$-acetylated sugars, and the cnidocytes responding by effecting nematocyst discharge (Thorington and Hessinger, 1988b; Watson and Hessinger, 1989). Like its predecessor, this model permits the nervous system to modulate the overall responsiveness of the CSCCs.

In this study, we present evidence that neither cnidocytes alone nor individual CSCCs are independent effectors of nematocyst discharge. Comparison of densities of BFCs and nematocysts discharged, both with and without chemosensitization, indicates that the ratio of BFCs to responding CSCCs is approximately 1 to 3 when only microbasic p-mastigophore nematocysts are considered and approximately 1 to 7 when all types of responding CSCCs are included (see Materials and Methods). We propose that relatively few cells, the BFCs, are somehow involved with CSCCs in regulating the discharge of nematocysts.

\section{Conclusion}

We have shown that epidermal cells exhibiting relatively high $\mathrm{Ca}^{2+}$ levels occur in anemone tentacles both with and without chemosensitization. However, when chemoreceptors for $\mathrm{N}$-acetylated sugars are activated, the number of cells that exhibit the highest $\mathrm{Ca}^{2+}$ levels increases two- to threefold-an enhancement comparable to that seen in nematocyst discharge upon chemosensitization. Timecourses depicting the abundance of these cells upon mucin treatment correlate to timecourses both for nematocyst discharge to mucin and for supportingcell chemoreceptor labeling. The scarcity of the cells exhibiting the highest $\mathrm{Ca}^{2+}$ levels indicates that not cvery activated CSCC, if any, participates in this intense $\mathrm{Ca}^{2+}$ signaling. Although the identity of the cells exhibiting the highest $\mathrm{Ca}^{2+}$ levels is frequently uncertain, it appears that these few cells, using $\mathrm{Ca}^{2+}$ as a second messenger, are somehow involved in regulating discharge of nematocysts from many cnidocytes. This regulation occurs via specific remote control, either with or without some local control by individual CSCCs. Thus, the evidence presented here suggests a major departure from the independent effector hypothesis explaining regulation of nematocyst discharge in sea anemones.

\section{Acknowledgments}

We are grateful to Dr. Paul G. Greenwood for critically reading an earlier version of the manuscript. This work was supported by NSF DCB-9105058 and LEQSF RDA44 and GF-19.

\section{Literature Cited}

Bigger, C. H. 1982. The cellular basis of the aggressive acrorhagial response of sea anemones. J. Morph. 173: 259-278.

Boothby, K. M., and I. D. McFarlane. 1986. Chemoreception in sea anemones: Betaine stimulates the pre-feeding response in Urticina eques and $U$. felina. J. Exp. Biol. 125: 385-389.

Bullock, T. H., and G. A. Horridge. 1965. Structure and Function in the Nervous System of Invertebrates, Vol. 1. W. H. Freeman and Co., San Francisco. 798 pp.

Conklin, E. J., and R. N. Mariscal. 1976. Increase in nematocyst and spirocyst discharge in a sea anemone in response to mechanical stimulation. Pp. 549-558 in Coelenterate Ecology and Behavior, G. O. Mackie, ed. Plenum Press, New York.

Ewer, R. F. 1947. On the functions and mode of action of the nematocysts of hydra. Proc. Zool. Soc. Lond. 117: 365-376.

Grimmelikhuijzen, C. J. P., D. Graff, O. Koizumi, J. A. Westfall, and I. D. McFarlane. 1990. Neurons and their peptide transmitters in coelenterates. Pp. 95-109 in Evolution of the First Nervous Systems, P. A. V. Anderson, ed. Plenum Press, New York.

Holstein, T., and P. Tardent. 1984. An ultrahigh-speed analysis of exocytosis: nematocyst discharge. Science 223: 830-833.

Lubbock, R. 1979. Chemical recognition and nematocyte excitation in a sea anemone. J. Exp. Biol. 83: 283-292.

Mariscal, R. N. 1974. Nematocysts. Pp. 129-178 in Coelenterate Biology, Reviews and New Perspectives, L. Muscatine and H. M. Lenhoff, eds. Academic Press, New York.

Mariscal, R. N., E. J. Conklin, and C. H. Bigger. 1978. The putative sensory receptors associated with the cnidae of cnidarians. Scanning Electron Microsc. 2: 959-966.

McFarlane, I. D., D. Graff, and C. J. P. Grimmelikhuijzen. 1990. Peptidergic neurotransmitters in the anthozoa. Pp. 111-127 in Evolution of the First Nervous Systems, P. A. V. Anderson, ed. Plenum Press, New York.

Minta, A., J. Kao, and R. Tsien. 1989. Fluorescent indicators for cytosolic calcium based on rhodamine and fluorescein chromophores. J. Biol. Chem. 264: 8171-8178.

Pantin, C. F. A. 1942a. Excitation of nematocysts. Nature 149: 109.

Pantin, C. F. A. 1942b. The excitation of nematocysts. J. Exp. Biol. 19: 294-310.

Parker, G. H. 1916. The effector system of actinians. J. Exp. Zool. 21: 461-484

Parker, G. H. 1919. The Elementary Nervous System, Lippincott, Philadelphia.

Parker, G. H., and M. A. Van Alstyne. 1932. The control and discharge of nematocysts, especially in Metridium and Physalia. J. Exp. Biol. 63: 329-344.

Peteya, D. J. 1975. The ciliary-cone sensory cell of anemones and cerianthids. Tissue Cell 7(2): 243-252.

Robson, E. A. 1988. Problems of supply and demand for cnidae in anthozoa. Pp. 179-207 in The Biology of Nematocysts, D. A. Hessinger and H. M. Lenhoff, eds. Academic Press, San Diego.

Shelton, G. A. B. 1982. Anthozoa. Pp. 203-242 in Electrical Conduction and Behaviour in "Simple" Invertebrates, G. A. B. Shelton, ed. Oxford University Press, New York.

Skaer, R. J., and L. E. R. Picken. 1965. The structure of the nematocyst thread and the geometry of discharge in Corynactis viridis (Allman). Phil. Trans. R. Soc. Lond., Ser. B 250: 131-164. 
Thibodeaux, P. M., and G. M. Watson. 1991. Imaging of intracellular calcium fluxes in tentacles of a sea anemone. Am. Zool. 31: 15a.

Thorington, G. U., and D. A. Hessinger. 1988a. Control of cnida discharge: I. Evidence for two classes of chemoreceptor. Biol. Bull. 174: 163-171.

Thorington, G. U., and D. A. I Iessinger. 1988b. Control of discharge: factors affecting discharge of cnidae. Pp. 233-253 in The Biology of Nematocysts, D. A. Hessinger and H. M. Lenhoff, eds. Academic Press, San Diego.

Thorington, G. U., and D. A. Hessinger. 1990. Control of cnida discharge III: Spirocysts are regulated by three classes of chemoreceptors. Biol. Bull. 178: 74-83.

van Marle, J. 1990. Catecholamines, related compounds and the nervous system in the tentacles of some anthozoans. Pp. 129-140 in Evolution of the First Nervous Systems, P. A. V. Anderson, ed. Plenum Press, New York.
Watson, G. M., and D. A. Hessinger. 1987. Receptor-mediated endocytosis of a chemoreceptor involved in triggering the discharge of cnidae in a sea anemone tentacle. Tissue Cell 19: 747-755.

Watson, G. M., and D. A. Hessinger. 1989. Cnidocytes and adjacent supporting cells form receptor-effector complexes in anemone tentacles. Tissue Cell 21: 17-24.

Westfall, J. A. 1973. Ultrastructural evidence for neuromuscular systems in coelenterates. Am. Zool. 13: 237-246.

Westfall, J. A. 1988. Presumed neuronematocyte synapses and possible pathways controlling discharge of a battery of nematocysts in Hydra. Pp. 41-51 in The Biology of Nematocysts, D. A. Hessinger and H. M. Lenhoff, eds. Academic Press, San Diego.

Williams, R. B. 1968. Control of the discharge of cnidae in Diadumene luciae (Verrill). Nature 219: 959. 